\title{
Multiplicity of normalized solutions for p-Laplacian equation with critical growth in $\mathrm{RN}$
}

\author{
Xueqin Peng ${ }^{1}$ \\ ${ }^{1}$ University of Shanghai for Science and Technology
}

June 2, 2021

\begin{abstract}
In this paper, we consider the following p-Laplacian equation ? ? ? ? ? - ? $] \mathrm{pu}+|\mathrm{u}| \mathrm{p}-2 \mathrm{u}-\lambda \mathrm{u}=\mu|\mathrm{u}| \mathrm{q}-2 \mathrm{u}+|\mathrm{u}| \mathrm{p}^{*}-2 \mathrm{u}, \mathrm{in} \mathrm{RN}, \mathrm{u}$ $>0$, [?] $\mathrm{RNu} 2 \mathrm{dx}=\mathrm{a} 2$, where $\mathrm{a}, \mu>0,-[?] \mathrm{pu}=\operatorname{div}(|[?] \mathrm{u}| \mathrm{p}-2[?] \mathrm{u}), 1<\mathrm{p}<\mathrm{N}, \lambda[?] \mathrm{R}$ is an unknown parameter that appears as a Lagrange multiplier, $\mathrm{p}<\mathrm{q}$
\end{abstract}

\section{Hosted file}

P-Multiplicity-NS.pdf available at https://authorea.com/users/417526/articles/524598multiplicity-of-normalized-solutions-for-p-laplacian-equation-with-critical-growth-in-rn 